MMCA-97 Conference, pp. 66-74

R. Čiegis (Ed)

(C) 1997 Vilniaus Gedimino technikos universitetas

\title{
THE CONVERGENCE AND STABILITY OF EXPLICIT DIFFERENCE SCHEMES FOR NONLINEAR SCHRÖDINGER EQUATION
}

\author{
F. IVANAUSKAS and M. RADŽIŪNAS \\ Faculty of Mathematics, Vilnius University, \\ Naugarduko 24, Vilnius 2006, Lithuania
}

\section{INTRODUCTION}

We consider the first boundary value problem for a nonlinear Schrödinger type equation. There are a lot of studies on the numerical solution of initial and initial-boundary problems for such equations [1-4]. We are interested in finite difference methods, that have some grid analogues of conservation laws. A three-layered explicit difference scheme of DuFort-Frankel-type is explicit, conditionally stable and have some grid conservation properties. This scheme was introduced for the Schrödinger equation in $[3,4]$. The consistency of this scheme requires the condition $\tau / h \rightarrow 0$, where $\tau$ and $h$ are time and space grid steps. In [4] the linear Schrödinger equations were investigated and stability of the schemes was proved. In [3] nonlinear equations were also discussed and the grid analogue of the conservative law in the space $L_{2}$ was obtained. But there were no proof of the convergence and stability of the difference scheme. Thus, our paper is developing the results of [3,4]. In the case of cubic nonlinearity we have obtained the analogues of conservation laws in the spaces $L_{2}$ and $W_{2}^{1}$. In the more general case we have got a new type of $a$ priori estimates, which where also derived for two - layered schemes in $[1,2]$. Under the condition $\tau / h^{2} \leq \nu<1 / 2|a|$, where $a$ is the constant from the equation and $\nu$ is some arbitrary constant, the convergence and stability of the difference scheme in the norms of spaces $C$ and $W_{2}^{1}$ were proved.

\section{STATEMENT OF THE PROBLEM. AUXILIARY STATE- MENTS}

We consider the first initial-boundary value problem for the cubic Schrödinger equation

$$
\frac{\partial u}{\partial t}=i a \frac{\partial^{2} u}{\partial x^{2}}-i \lambda|u|^{2} u, \quad(x, t) \in Q
$$


with initial and boundary conditions

$$
u(0, t)=u(1, t)=0, \quad t \in[0 ; T], \quad u(x, 0)=u_{0}(x), \quad x \in[0 ; 1] .
$$

Here $i=\sqrt{-1}, Q=(0 ; 1) \times(0 ; T), a, \lambda$ are real constants, $a \neq 0 ; u(x, t)$ is a complex-valued function.

Note, that in d-dimensional case we can consider the equation

$$
\frac{\partial u}{\partial t}=i a \sum_{j=1}^{d} \frac{\partial^{2} u}{\partial x_{j}^{2}}-i \lambda|u|^{2} u
$$

instead of the equation (2.1).

We use usual definitions of the inner product in the space $L_{2}$ and the norms of the complex-valued functions in the Sobolev spaces $L_{p}$ and $W_{2}^{1}$ as

$$
(v, w)=\int_{0}^{1} v(x) w^{*}(x) d x,\|v\|_{L_{p}}^{p}=\int_{0}^{1}|v(x)|^{p} d x,\|v\|_{W_{2}^{1}}^{2}=\|v\|_{L_{2}}^{2}+\left\|\frac{\partial v}{\partial x}\right\|_{L_{2}}^{2} .
$$

Here $w^{*}(x)$ is the complex conjugate of $w(x)$. Also we define $\operatorname{Re} v$ and $\operatorname{Im} v$ as real and imaginary parts of the complex expression $v$. Let $C(\bar{Q})$ also be the space of continuous functions with the norm $\|v\|_{C(\bar{Q})}=\max _{(x, t) \in \bar{Q}}|v(x, t)|$.

It is well-known, that the solution of the problem (2.1), (2.2) satisfies the following conservation laws for all $t \in[0, T]$ :

$$
\begin{gathered}
\|u(t)\|_{L_{2}}=I_{1}(t)=I_{1}(0), \\
\left\|\frac{\partial u}{\partial x}(t)\right\|_{L_{2}}^{2}+(\lambda / 2 a)\|u(t)\|_{L_{4}}^{4}=I_{2}(t)=I_{2}(0) .
\end{gathered}
$$

Note, that these laws are also satisfied in d-dimensional case, where $d \geq 1$.

We introduce a uniform grid with steps $\tau$ and $h$ in the domain $\bar{Q}: \bar{Q}_{h}=\bar{\omega}_{h} \times$ $\bar{\omega}_{\tau}$ and $Q_{h}=\omega_{h} \times \omega_{\tau}$. We consider that $\tau=T / M, t_{j}=j \tau, h=1 / N, x_{l}=l h$, $\bar{\omega}_{\tau}=\left\{t_{j} ; j=0, \ldots, M\right\}, \omega_{\tau}=\left\{t_{j} ; j=1, \ldots, M-1\right\}, \bar{\omega}_{h}=\left\{x_{l} ; l=0, \ldots, N\right\}$, $\omega_{h}=\left\{x_{l} ; l=1, \ldots, N-1\right\}$.

We shall use grid the analogues of the Sobolev spaces $L_{p h}$ and $W_{2 h}^{1} \cdot C_{h}$ denotes the analogue of the space $C(\bar{Q})$. Let us define inner products at the grid $\bar{\omega}_{h},(u, v)=\sum_{l=1}^{N-1} u_{l} v_{l}^{*} h,(u, v]=\sum_{l=1}^{N} u_{l} v_{l}^{*} h$. The norms in this grid are defined as follows:

$$
\left.\left.\|u\|_{L_{p h}}^{p}=\sum_{l=1}^{N-1}\left|u_{l}\right|^{p} h, \| u\right]\left.\right|^{2}=(u, u],\|u\|^{2}=(u, u),\|u\|_{W_{2 h}^{1}}^{2}=\|u\|^{2}+\| u_{\bar{x}}\right]\left.\right|^{2} .
$$

In d-dimensional cases we can use analogous notations. For example, 2dimensional norms and inner products can be written in a following way: $(u, v)=\sum_{l, k=1}^{N-1} u_{l, k} v_{l, k}^{*} h^{2},(u, v]=\sum_{l, k=1}^{N} u_{l, k} v_{l, k}^{*} h^{2},\|u\|_{L_{p h}}^{p}=\sum_{l, k=1}^{N-1}\left|u_{l, k}\right|^{p} h^{2}$. 
We denote $p=p_{l}^{j}=p\left(x_{l}, t_{j}\right), \hat{p}=p_{l}^{j+1}, \check{p}=p_{l}^{j-1}, \dot{p}=(\check{p}+\hat{p}) / 2, p_{+}=p_{l+1}^{j}$, $p_{-}=p_{l-1}^{j}, \bar{p}=p_{-}+p_{+}, p_{t}=(\hat{p}-\check{p}) / 2 \tau, p_{\bar{x}}=\left(p-p_{-}\right) / h, p_{x \nwarrow}=\left(\check{p}-p_{-}\right) / h$, $p_{x \swarrow}=\left(p-\check{p}_{-}\right) / h$.

In d-dimensional cases we can use analogous notations. For example, 2dimensional notations can be written as follows: $p=p_{l, k}^{j}=p\left(x_{l}, y_{k}, t_{j}\right)$, $p_{\cdot,+}=p_{l, k+1}^{j}, p_{\cdot,-}=p_{l, k-1}^{j}, p_{+, \cdot}=p_{l+1, k}^{j}, p_{-,,}=p_{l-1, k}^{j}, \bar{p}=p_{\cdot,-}+p_{\cdot,+}+$ $p_{-, \cdot}+p_{+, \cdot,}, p_{x \nwarrow}=\left(\check{p}-p_{-, .}\right) / h, p_{x_{\swarrow}}=\left(p-\check{p}_{-, .}\right) / h . p_{y \nwarrow}=\left(\check{p}-p_{\cdot,-}\right) / h$, $p_{y \swarrow}=\left(p-\check{p}_{,+}\right) / h$.

We can prove the following grid analogues of one imbedding theorem and a multiplicative inequality:

Let $v_{0}=\hat{v}_{0}=v_{N}=\hat{v}_{N}=0$. Then

$$
\begin{gathered}
\left.\left.\max \left\{\|\hat{v}\|_{C_{h}},\|v\|_{C_{h}}\right\} \leq 0.5\left(\| \hat{v}_{x \nwarrow}\right] \mid+\| \hat{v}_{x_{\swarrow}}\right]\right), \\
\left.\left.\max \left\{\|\hat{v}\|_{C_{h}}^{2},\|v\|_{C_{h}}^{2}\right\} \leq 0.5(\|\hat{v}\|+\|v\|)\left(\| \hat{v}_{x \nwarrow}\right] \mid+\| \hat{v}_{x_{\swarrow}}\right] \mid\right) .
\end{gathered}
$$

In the following we shall also use a grid analogue of the Gronwall inequality and some well-known imbedding theorems for the grid functions $v, v_{0}=v_{N}=$ 0 :

$$
\left.\|v\|_{L_{p h}} \leq\|v\|_{C_{h}} \leq 0.5 \| v_{\bar{x}}\right] \mid \leq 0.5\|v\|_{W_{2 h}^{1}}
$$

\section{THE DIFFERENCE SCHEME. GRID CONSERVATION LAWS}

We relate the problem (2.1), (2.2) with the following DuFort-Frankel type difference scheme:

$$
\begin{gathered}
p_{t}=i a \frac{\bar{p}-2 \dot{p}}{h^{2}}-i \lambda|p|^{2} \dot{p}, \quad(x, t) \in Q_{h}, \\
p\left(x_{0}, t\right)=p\left(x_{N}, t\right)=0, \quad t \in \bar{\omega}_{\tau}, \quad p(x, 0)=u_{0}(x), \quad x \in \bar{\omega}_{h} .
\end{gathered}
$$

The solution on the first layer $t_{1}$ can be found using some two-layered scheme.

Note, that in d-dimensional case instead of (3.1) scheme we shall have

$$
p_{t}=i a \frac{\bar{p}-4 \dot{p}}{h^{2}}-i \lambda|p|^{2} \dot{p}
$$

In [3] one case of a grid analogue of (2.3) for the difference scheme (3.1), (3.2) was investigated. We can prove the following grid analogues of (2.3) and (2.4):

Lemma 3.1. [Grid analogue of (2.3)] The equality

$$
\left\|p\left(t_{j+1}\right)\right\|^{2}+\left\|p\left(t_{j}\right)\right\|^{2}+\frac{2 a \tau}{h^{2}} \operatorname{Im}\left(\bar{p}\left(t_{j}\right), p\left(t_{j+1}\right)\right)=I_{1 h}\left(t_{j}\right)=I_{1 h}\left(t_{0}\right)
$$


is valid for the solution of difference scheme (3.1), (3.2) for all $j=0, \ldots, M-$ 1. Let the condition on the ratio of time and space grid steps

$$
0<\frac{2|a| \tau}{h^{2}} \leq \nu<1
$$

where $\nu$ is arbitrary constant, be satisfied. Then the following estimate is valid:

$$
\left\|p\left(t_{j+1}\right)\right\|^{2}+\left\|p\left(t_{j}\right)\right\|^{2} \leq \mu\left(\left\|p\left(t_{1}\right)\right\|^{2}+\left\|p\left(t_{0}\right)\right\|^{2}\right) .
$$

Here and later $\mu=\frac{1+\nu}{1-\nu}$.

Lemma 3.2. [Grid analogue of (2.4)] The equality

$$
\left.\left.\| p_{x \nwarrow}\left(t_{j+1}\right)\right]\left.\right|^{2}+\| p_{x_{\swarrow}}\left(t_{j+1}\right)\right]\left.\right|^{2}+\frac{\lambda}{a}\left\|p\left(t_{j+1}\right) p\left(t_{j}\right)\right\|^{2}=I_{2 h}\left(t_{j}\right)=I_{2 h}\left(t_{0}\right)
$$

is valid for the solution of the scheme (3.1), (3.2) for all $j=0, \ldots, M-1$.

Remark. Note, that in d-dimensional case we can derive analogous grid conservative laws. For example, in 2-dimensional case the first law reads in the same way as (3.3), and the second law is

$$
\begin{gathered}
\left.\left.\left.\left.\| p_{x \nwarrow}\left(t_{j+1}\right)\right]\left.\right|^{2}+\| p_{x_{\swarrow}}\left(t_{j+1}\right)\right]\left.\right|^{2}+\| p_{y \nwarrow}\left(t_{j+1}\right)\right]\left.\right|^{2}+\| p_{y_{\swarrow}}\left(t_{j+1}\right)\right]\left.\right|^{2} \\
+\frac{\lambda}{a}\left\|p\left(t_{j+1}\right) p\left(t_{j}\right)\right\|^{2}=I_{2 h}\left(t_{j}\right)=I_{2 h}\left(t_{0}\right) .
\end{gathered}
$$

\section{CONVERGENCE AND STABILITY OF THE DIFFERENCE SCHEME}

Suppose that the solution of the problem (2.1), (2.2) is smooth enough to satisfy the approximation of the difference scheme. Let $\Phi\left(t_{j}\right)$ be a truncation error. It is easy to find that this error is of order $O\left(\tau^{2}+h^{2}+(\tau / h)^{2}\right)$. Thus, the consistency of the scheme requires the condition $\tau / h \rightarrow 0$ to be fulfilled.

Suppose that the solution of the problem (2.1), (2.2) is also smooth enough to satisfy the following conditions:

$$
\max _{t_{j} \in \omega_{\tau}}\left\{\left\|\Phi\left(t_{j}\right)\right\|_{L_{2 h}}\right\} \rightarrow 0, \quad \tau, h \rightarrow 0
$$

and

$$
M_{1}=\max _{t \in[0, T]}\|u(t)\|_{W_{2}^{1}}<\infty, \quad M_{2}=\max _{t \in[0, T]}\left\|\frac{\partial u}{\partial t}(t)\right\|_{L_{2}}<\infty
$$


¿From here and from the imbedding theorem $\stackrel{\circ}{W}_{2}^{1} \rightarrow C$ it follows that

$$
\max _{t \in[0, T]}\|u(t)\|_{L_{2}} \leq\|u\|_{C(\bar{Q})} \leq 0.5 M_{1} .
$$

Let $\varepsilon=u-p$ be an error of the solution. Then we have the following difference scheme for this error:

$$
\begin{gathered}
\varepsilon_{t}=\frac{i a}{h^{2}}(\bar{\varepsilon}-2 \dot{\varepsilon})+\Psi+\Phi, \quad(x, t) \in Q_{h}, \\
\varepsilon(x, 0)=0 \quad x \in \bar{\omega}_{h}, \quad \varepsilon\left(x_{0}, t\right)=\varepsilon\left(x_{N}, t\right)=0 \quad t \in \bar{\omega}_{\tau} .
\end{gathered}
$$

Here

$$
\Psi=-i \lambda\left(|u|^{2} \dot{u}-|p|^{2} \dot{p}\right) .
$$

Suppose that the function $\varepsilon$ on the first layer satisfies the condition

$$
\left\|\varepsilon\left(t_{1}\right)\right\|_{W_{2 h}^{1}}^{2} \rightarrow 0, \quad \tau, h \rightarrow 0
$$

Noting, that the equality

$$
\left.\left.\left.\left.\| \hat{p}_{x \nwarrow}\right]\left.\right|^{2}+\| \hat{p}_{x_{\swarrow}}\right]\left.\right|^{2}=\| \hat{p}_{\bar{x}}\right]\left.\right|^{2}+\| p_{\bar{x}}\right]\left.\right|^{2}+\frac{2 \tau^{2}}{h^{2}} \operatorname{Re}\left(\frac{\hat{p}-p}{\tau}, \frac{\hat{p}_{-}-p_{-}}{\tau}\right)
$$

is valid, we can prove one more auxiliary lemma.

Lemma 4.1. Suppose that the conditions (3.4) and (4.6) are satisfied. Then there exist constants $\tau_{0}>0$ and $h_{0}>0$ such that for all positive $\tau \leq \tau_{0}$ and $h \leq h_{0}$ the following estimates for the solution of the problem (3.1), (3.2) are valid:

$$
\begin{gathered}
\left.\left.\left.\max _{1 \leq j \leq M} \| p_{x \nwarrow}\left(t_{j}\right)\right] \mid+\| p_{x_{\swarrow}}\left(t_{j}\right)\right] \mid\right) \leq M_{3}, \\
\|p\|_{C\left(\bar{Q}_{h}\right)}=\max _{\left(x_{l}, t_{j}\right) \in \bar{Q}_{h}}\left|p\left(x_{l}, t_{j}\right)\right| \leq 0.5 M_{3} .
\end{gathered}
$$

Here $M_{3}=M_{3}\left(a, \lambda, M_{1}, \nu\right)$.

Now, using Lemmas and other properties mentioned above, we can prove the convergence of the difference scheme in $L_{2}$ and $C$ norm.

Theorem 4.1. Let the conditions (3.4), (4.1), (4.2), (4.6) be satisfied. Then the solution of the problem (3.1), (3.2) converges to the solution of the problem (2.1), (2.2) in the spaces $L_{2}$ and $C\left(\bar{Q}_{h}\right)$. There exist constants $\tau_{0}>0$ and $h_{0}>0$ such that for all positive $\tau \leq \tau_{0}$ and $h \leq h_{0}$ the following estimates are valid:

$$
\|\varepsilon\| \leq c_{1}\left\|\varepsilon\left(t_{1}\right)\right\|+c_{2} \max _{1 \leq j \leq M-1}\left\{\left\|\Phi\left(t_{j}\right)\right\|\right\}
$$




$$
\|\varepsilon\|_{C\left(\bar{Q}_{h}\right)}^{2} \leq c_{3}\left\|\varepsilon\left(t_{1}\right)\right\|+c_{4} \max _{1 \leq j \leq M-1}\left\{\left\|\Phi\left(t_{j}\right)\right\|\right\}
$$

Here $c_{l}=c_{l}\left(a, \lambda, \nu, M_{1}, T\right), l=1, \ldots, 4$.

We can also prove the stability of the difference scheme on initial data in the same norms.

Let $u_{1}(x, t), u_{2}(x, t)$ and $p_{1}, p_{2}$ be the solutions of the problems $(2.1),(2.2)$ and $(3.1),(3.2)$ with the initial data $u_{10}(x)$ and $u_{20}(x)$ respectively.

THEOREM 4.2. Let the conditions of Theorem 4.1 be satisfied. Then there exist constants $\tau_{0}>0$ and $h_{0}>0$ such that for all positive $\tau \leq \tau_{0}$ and $h \leq h_{0}$ the following estimates hold:

$$
\begin{gathered}
\left\|p_{1}-p_{2}\right\| \leq c_{5}\left\|u_{10}-u_{20}\right\| . \\
\left\|p_{1}-p_{2}\right\|_{C\left(\bar{Q}_{h}\right)}^{2} \leq c_{6}\left\|u_{10}-u_{20}\right\| .
\end{gathered}
$$

Here $c_{l}=c_{l}\left(a, \lambda, \nu, T, \max _{t \in[T ; 0]}\left\{\left\|u_{1}(t)\right\|_{W_{2}^{1}},\left\|u_{2}(t)\right\|_{W_{2}^{1}}\right\}\right), l=5,6$.

\section{A GENERAL CASE OF THE PROBLEM}

We consider the nonlinear Schrödinger equation

$$
\frac{\partial u}{\partial t}=i a \frac{\partial^{2} u}{\partial x^{2}}+f\left(u, u^{*}\right) u
$$

Here $f\left(u, u^{*}\right)$ is a polynomial with arguments $u$ and $u^{*}, f\left(u, u^{*}\right)=f\left(-u,-u^{*}\right)$. We can find a continuous nondecreasing function $\varphi(y)$ that satisfies the conditions

$$
\left|f\left(u, u^{*}\right)\right| \leq \varphi(|u|), \quad\left|D^{\mathbf{j}} f\left(u, u^{*}\right) u\right| \leq \varphi(|u|), \quad|\mathbf{j}|=1,2
$$

here $\mathbf{j}$ is a two-dimensional vector, $|\mathbf{j}|=j_{1}+j_{2}, D^{\mathbf{j}}=\partial^{|\mathbf{j}|} / \partial u^{j_{1}} \partial u^{* j_{2}}$.

We relate the equation (5.1) with the following difference scheme:

$$
p_{t}=i a \frac{\bar{p}-2 \dot{p}}{h^{2}}+f\left(p, p^{*}\right) \dot{p}, \quad(x, t) \in Q_{h}
$$

It can be proved that the following estimates for the nonlinear grid function $f\left(v, v^{*}\right) \dot{v}$ are satisfied:

$$
\begin{gathered}
\left|\left(f\left(v, v^{*}\right) \dot{v}, \dot{v}\right)\right| \leq 0.5 \varphi\left(\|v\|_{C_{h}}\right)\left(\|\hat{v}\|^{2}+\|\check{v}\|^{2}\right) \\
\left|\left(f\left(v, v^{*}\right) \dot{v}-f\left(w, w^{*}\right) \dot{w}, \dot{v}-\dot{w}\right)\right| \leq \varphi\left(\max \left\{\|\check{v}\|_{C_{h}},\|v\|_{C_{h}},\|\hat{v}\|_{C_{h}},\|w\|_{C_{h}}\right\}\right)
\end{gathered}
$$




$$
\times\left(\|\check{v}-\check{w}\|^{2}+\|v-w\|^{2}+\|\hat{v}-\hat{w}\|^{2}\right) .
$$

From (5.5) we can obtain

$$
\left.\left.\left.\left|\left(\left(f\left(v, v^{*}\right) \dot{v}\right)_{\bar{x}}, \dot{v}_{\bar{x}}\right]\right| \leq\left.\varphi\left(\max \left\{\|\check{v}\|_{C_{h}},\|v\|_{C_{h}},\|\hat{v}\|_{C_{h}}\right\}\right)\left(\| \check{v}_{\bar{x}}\right]\right|^{2}+\| v_{\bar{x}}\right]\left.\right|^{2}+\| \hat{v}_{\bar{x}}\right]\left.\right|^{2}\right) .
$$

Also we have

$$
\begin{aligned}
& \left.\left.\left|\left(\left(f\left(v, v^{*}\right) \dot{v}\left(t_{k}\right)-f\left(w, w^{*}\right) \dot{w}\left(t_{k}\right)\right)_{\bar{x}}, \dot{z}_{\bar{x}}\left(t_{k}\right)\right]\right| \leq\left(\max _{l=-1,0,1}\left\{1, \| v_{\bar{x}}\left(t_{k+l}\right)\right] \mid, \| w_{\bar{x}}\left(t_{k+l}\right)\right] \mid\right\}\right) \\
& \left.\quad \times c \varphi\left(\max _{l=-1,0,1}\left\{\left\|v\left(t_{k+l}\right)\right\|_{C_{h}},\left\|w\left(t_{k+l}\right)\right\|_{C_{h}}\right\}\right)\left(\left.\max _{l=-1,0,1}\left\{\| z_{\bar{x}}\left(t_{k+l}\right)\right]\right|^{2}\right\}\right)
\end{aligned}
$$

where $c$ is some constant and $z=v-w$.

One can prove the estimates (5.4)-(5.7) in an analogous manner as the similar estimates in [2].

Proving the convergence and stability of scheme we obtain and use a new type of a priori estimates [1,2], instead of equalities of the type (3.3) or (3.6).

Let $p$ be the solution of the difference scheme (5.3), (3.2). We denote the fictitious nodes of the grid $(-h, \tau j)$ and $(1+h, \tau j)$, where $j=0, \ldots, M$. Let $v_{-1}$ and $v_{N+1}$ be the values of grid function on these nodes. We define the solution of the difference scheme on these nodes as follows: $p_{-1}=-p_{1}$ and $p_{N+1}=-p_{N-1}$. This corresponds to the boundary conditions (2.2) and to the equality $\frac{\partial^{2} u}{\partial x^{2}}(0, t)=\frac{\partial^{2} u}{\partial x^{2}}(1, t)=0$. Here $u$ is the solution of the extended differential problem (5.1), (2.2) on the frontier of the domain. The extension is valid due to the zero boundary conditions and since the nonlinear function is odd.

Assume also that the truncation error satisfies the condition

$$
\max _{t_{j} \in \omega_{\tau}}\left\{\left\|\Phi\left(t_{j}\right)\right\|_{W_{2 h}^{1}}\right\} \rightarrow 0, \quad \tau, h \rightarrow 0
$$

An error of the solution of the problem (5.1), (2.2) satisfies the equalities (4.4), (4.5) where

$$
\Psi=\left(f\left(u, u^{*}\right) \dot{u}-f\left(p, p^{*}\right) \dot{p}\right) .
$$

We can prove now the convergence of the difference scheme (5.3), (3.2).

Theorem 5.1. Let the conditions (3.4), (4.2), (4.3), (4.6), (5.8) be satisfied. Then the solution of the difference scheme (5.3), (3.2) converges to the solution of the problem (5.1), (2.2) in spaces $W_{2 h}^{1}$ and $C\left(\bar{Q}_{h}\right)$. There exist constants $\tau_{0}>0$ and $h_{0}>0$ such that for all positive $\tau \leq \tau_{0}$ and $h \leq h_{0}$ the following estimates hold:

$$
\max _{t_{j} \in \bar{\omega}_{\tau}}\left\{\left\|\varepsilon\left(t_{j}\right)\right\|_{W_{2 h}^{1}}\right\} \leq c_{7}\left\|\varepsilon\left(t_{1}\right)\right\|_{W_{2 h}^{1}}+c_{8} \max _{t_{j} \in \omega_{\tau}}\left\{\left\|\Phi\left(t_{j}\right)\right\|_{W_{2 h}^{1}}\right\}
$$




$$
\|\varepsilon\|_{C\left(\bar{Q}_{h}\right)} \leq 0.5 c_{7}\left\|\varepsilon\left(t_{1}\right)\right\|_{W_{2 h}^{1}}+0.5 c_{8} \max _{t_{j} \in \omega_{\tau}}\left\{\left\|\Phi\left(t_{j}\right)\right\|_{W_{2 h}^{1}}\right\} ;
$$

here $c_{l}=c_{l}\left(a, \varphi, \nu, M_{1}, T\right), l=7,8$.

Similarly as in $[1,2]$, we prove the stability of the difference scheme on initial data. Let $u_{1}(x, t), u_{2}(x, t)$ and $p_{1}, p_{2}$ be the solutions of the problems (5.1), $(2.2)$ and (5.3), (3.2) with the initial data $u_{10}(x)$ and $u_{20}(x)$ respectively.

TheOREm 5.2. Let the conditions of Theorem 5.1 be satisfied. Then there exist constants $\tau_{0}>0$ and $h_{0}>0$ such that for all positive $\tau \leq \tau_{0}$ and $h \leq h_{0}$ the following estimates hold:

$$
\begin{gathered}
\max _{t_{j} \in \bar{\omega}_{\tau}}\left\{\left\|p_{1}\left(t_{j}\right)-p_{2}\left(t_{j}\right)\right\|_{W_{2 h}^{1}}\right\} \leq c_{9}\left\|u_{10}-u_{20}\right\|_{W_{2 h}^{1}}, \\
\left\|p_{1}-p_{2}\right\|_{C\left(\bar{Q}_{h}\right)} \leq 0.5 c_{9}\left\|u_{10}-u_{20}\right\|_{W_{2 h}^{1}} . \\
\text { Here } c_{9}=c_{9}\left(a, \varphi, \nu, T, \max _{t \in[0 ; T]}\left\{\left\|u_{1}(t)\right\|_{W_{2}^{1}},\left\|u_{2}(t)\right\|_{W_{2}^{1}}\right\}\right) .
\end{gathered}
$$

\section{THE RESULTS OF COMPUTATIONAL EXPERIMENT}

We compared some different numerical methods for the solution of the first boundary value problem for Cubic Schrödinger equation. These methods are DuFort-Frankel finite difference scheme; the method of discretization of the equation in all space variables and solving the system of ordinary differential equations using fourth order Runge-Kutta method; the split-step pseudospectral method, where the nonlinear equation in each time step is splitted into two equations, one of them is nonlinear and has obvious analytical solution, the other is linear Schrödinger equation and is solved using Fast Fourier Transform.

We have made computational experiments for both one and two-dimensional cases. But, since we do not know any analytical solution of the first boundary value problem in two-dimensional case, here we present only the results for one-dimensional case. The programs were written in programming language FORTRAN 77, the calculations were made on workstation of UNIX type.

Here we consider the equation in the domain $(x, t) \in[-10,10] \times[0,1]$

$$
\frac{\partial u}{\partial t}=i \frac{\partial^{2} u}{\partial x^{2}}+2 i|u|^{2} u
$$

with initial conditions

$$
u(x, 0)=\operatorname{sech}(x)=2(\exp (x)+\exp (-x))^{-1} .
$$

The solution of this problem is the following function:

$$
u(x, t)=\operatorname{sech}(x) \exp i t
$$


Here we suppose, that this function on the bounds of the domain is rather close to zero (it is of order $2 \exp (-10) \approx 0.9 \times 10^{-4}$ ), and during the computational experiment we considered zero boundary conditions.

As the example of these calculations, here are presented some results for the solution at the time moment $t=1$ :

\begin{tabular}{|l||l|l|}
\hline Method & Maximal absolute error & Computational time (seconds) \\
\hline DuFort-Frankel & 0.00022321 & 101 \\
& 0.00005570 & 198 \\
\hline Runge-Kutta & 0.00026826 & 89 \\
& 0.00006688 & 175 \\
\hline Pseudo-spectral & 0.00018200 & 12 \\
& 0.00004664 & 22 \\
\hline
\end{tabular}

These experiments have shown, that the fastest is pseudospectral method. The other two methods have shown similar results.

Acknowledgment. M.Radžiūnas thanks the Institute of Mathematical Modelling at the Technical University of Denmark for hospitality and permission to perform all experiments on the computers of the Institute.

\section{REFERENCES}

[1] F. Ivanauskas. On Convergence of difference schemes for Nonlinear Schrödinger equations, the Kuramoto-Tsuzuki equation and reaction-diffusion type systems, Liet. Mat. Rink., 34, 1994, P. 32-51.

[2] M. Radžiūnas. On convergence and stability of difference schemes for nonlinear Schrödinger type equations, Liet. Mat. Rink., 36, 1996, P. 224-244.

[3] L. Wu. DuFort-Frankel-type methods for linear and nonlinear Schrödinger equations, SIAM J. Numer. Anal., 33, 1996, P. 1526-1533.

[4] W. Dai. An unconditionally stable three-level explicit difference schemes for the Schrödinger equation with a variable coefficient, SIAM J. Numer. Anal., 29, 1992, P. $174-181$. 